\title{
Effects of S-1 as a second-line chemotherapy for patients with relapsed pancreatic cancer
}

\author{
KEINOSUKE ISHIDO, YOSHIKAZU TOYOKI, DAISUKE KUDO, NORIHISA KIMURA, \\ DAISUKE YAMANA, TAKUYA MIURA, SHINJI TSUTSUMI, TAKAHIRO MUROYA, \\ TORU YOSHIKAWA, HIROSHI OGASAWARA, SHINNOSUKE YONAIYAMA, \\ SHUNJI NARUMI and KENICHI HAKAMADA
}

Department of Gastrointestinal Surgery, Hirosaki University Graduate School of Medicine, Hirosaki, Japan

Received April 18,2011; Accepted August 19, 2011

DOI: $10.3892 / 01.2011 .412$

\begin{abstract}
Adjuvant chemotherapy with gemcitabine is the standard treatment in Japan for patients who have undergone resection of pancreatic cancer. However, few reports have described suitable regimens for patients who present cancer relapse following adjuvant chemotherapy. In the present study, we retrospectively evaluated the efficacy and safety of S-1, an oral fluoropyrimidine derivative, as a second-line chemotherapy for patients who had suffered relapse of pancreatic cancer following adjuvant chemotherapy with gemcitabine. A total of 51 patients with pancreatic cancer suffered relapse after curative resection and subsequent adjuvant chemotherapy with gemcitabine at our institution. A group of 26 of these patients were administered S-1 orally twice daily after meals at a dose of $80 \mathrm{mg} / \mathrm{m}^{2}$ for body surface areas for 14 consecutive days, followed by a 7-day rest (S-1 group). The remaining 25 patients received no additional anticancer drugs other than continuation of gemcitabine (GEM/BSC group). During a median follow-up period of 35 months, a significant difference was observed in overall survival (OAS) between the S-1 group and the control group (median OAS, 20.9 vs. 13.7 months; $p=0.0157$, log-rank test). Furthermore, there was a significant inter-group difference in survival after relapse (SAR) (median SAR, 11.4 vs. 6.20 months; $\mathrm{p}=0.0025$, log-rank test). No increase in grade $3 / 4$ hematological and non-hematological toxicity was observed in the S-1 group. In conclusion, second-line chemotherapy using a combination of S-1 and adjuvant chemotherapy with gemcitabine may be an efficient and beneficial strategy for patients with relapsed pancreatic cancer.
\end{abstract}

Correspondence to: Dr Keinosuke Ishido, Department of Gastrointestinal Surgery, Hirosaki University Graduate School of Medicine, 5 Zaifu-cho, Hirosaki 036-8562, Japan

E-mail: kekemo110@yahoo.co.jp

Key words: second-line chemotherapy, S-1, gemcitabine, pancreatic cancer

\section{Introduction}

Pancreatic cancer is one of the most aggressive types of malignancy, with the majority of patients exhibiting surgically unresectable disease at the time of diagnosis (1). Surgical resection is the only potentially curative therapy, but even in resectable cases the overall 5-year survival rate is only $15-20 \%$ (2-3). Accordingly, surgical resection, as well as other forms of adjuvant therapy are required for improving the prognosis of such patients.

Since Neoptolemos et al reported the significant effect of postoperative chemotherapy on survival time after curative resection for pancreatic cancer (4), a number of studies have focused on adjuvant postoperative chemotherapy for improving the outcome of patients with pancreatic cancer (5-6). Gemcitabine (GEM), a deoxycytidine analogue of arabinosylcytosine, is one of the most promising chemotherapeutic agents to have emerged in recent years. Oettle et al reported that adjuvant chemotherapy with GEM was capable of prolonging not only disease-free survival, but also overall survival following curative resection for pancreatic cancer (7). That report, known as the CONKO-001 study, resulted in the adoption of GEM as a standard form of adjuvant chemotherapy following resection of pancreatic cancer. However, few reports have described suitable regimens for patients who suffer relapse after adjuvant chemotherapy.

Thus, we retrospectively evaluated the efficacy and safety of S-1, an oral fluoropyrimidine derivative (8), as a secondline chemotherapy for patients who had suffered relapse after adjuvant chemotherapy with GEM.

\section{Patients and methods}

Patients. Between 2001 and 2009, 51 patients with pancreatic cancer treated at our institution suffered relapse after curative resection and subsequent adjuvant chemotherapy with GEM. A group of 26 of these patients received S-1 orally twice daily after meals at a dose of $80 \mathrm{mg} / \mathrm{m}^{2}$ for body surface areas for 14 consecutive days, followed by a 7-day rest (S-1 group). After the disease was judged to be progressive, 10 patients underwent a third-line chemotherapy. In total, 3 patients were administered paclitaxel at 
Table I. Patient characteristics.

\begin{tabular}{|c|c|c|c|}
\hline & S-1 & GEM/BSC & p-value \\
\hline Patients & 26 & 25 & \\
\hline Gender (male/female) & $14 / 12$ & $14 / 11$ & 0.903 \\
\hline Age (years) & $63.8(50-78)$ & $68.4(48-81)$ & 0.091 \\
\hline $\begin{array}{l}\text { Pathological stage } \\
\text { (I/II/III/IV) }\end{array}$ & $6 / 18 / 0 / 2$ & $4 / 20 / 0 / 1$ & 0.416 \\
\hline $\mathrm{T}$ factor $(\mathrm{T} 1,2 / \mathrm{T} 3,4)$ & $3 / 23$ & $4 / 21$ & 0.406 \\
\hline $\mathrm{N}$ factor $(\mathrm{N} 0 / \mathrm{N} 1)$ & $9 / 17$ & $14 / 11$ & 0.125 \\
\hline $\begin{array}{l}\text { Operative procedure } \\
\text { (head/distal resection) }\end{array}$ & $20 / 6$ & $18 / 7$ & 0.938 \\
\hline Resection status (R0/R1) & $23 / 3$ & $22 / 3$ & 0.959 \\
\hline 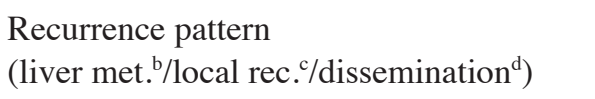 & $6 / 16 / 4$ & $8 / 13 / 4$ & 0.188 \\
\hline Median of disease-free survival (months) & 6.40 & 5.86 & 0.602 \\
\hline
\end{tabular}

GEM/BSC, gemcitabine group. ${ }^{a}$ UICC sixth edition; ${ }^{b}$ liver metastasis; 'local recurrence; ${ }^{\mathrm{d}}$ peritoneal dissemination.

$80 \mathrm{mg} / \mathrm{m}^{2} ; 4$ patients returned to chemotherapy with GEM (at 1000 or $800 \mathrm{mg} / \mathrm{m}^{2}$ ); 2 patients were administered GEM and S-1 concurrently; and 2 patients underwent the two-drug chemotherapy with CDDP and CPT-11. The remaining 25 patients were not administered any other anticancer drugs other than continuation of GEM (GEM/BSC group). If GEM was continued after disease recurrence, it was administered at $1000 \mathrm{mg} / \mathrm{m}^{2}$ bi-weekly for as long as possible. Among the latter 25 patients, 5 (20\%) continued to receive GEM, and 20 $(80 \%)$ were not administered any other anticancer drugs. The differences between the S-1 and GEM groups were analyzed with regard to patient demographics, clinical characteristics, overall survival (OAS), and survival after recurrence (SAR).

Statistical analysis. Demographic and clinical characteristics were expressed as means, medians and ranges (continuous outcomes). Groups were compared using the Wilcoxon rank-sum test for continuous outcomes and the Fisher's exact test for categorical outcomes. Survival distributions were estimated using the Kaplan-Meier method, and groups were compared using the log-rank test. Differences were considered to be significant at $p<0.05$. The data were analyzed using the Stat View software program (Abacus Concepts, Inc., Berkeley, California, USA).

\section{Results}

Patient characteristics. Patient characteristics in the S-1 and GEM/BSC groups are shown in Table I. This retrospective study included 51 patients (26 in the S-1 group and 25 in the GEM/BSC group). The following parameters were compared between the groups: gender, age, final stage, $\mathrm{T}$ factor, $\mathrm{N}$ factor, operative procedure employed, R0/R1 resection rate, and pattern of recurrence. However, the two groups were statistically similar. Disease-free survival periods for the two groups were estimated by the Kaplan-Meier method. The median disease-free survival period was 6.4 months in the S-1 group and 5.9 months in the GEM/BSC group; the difference was not significant $(\mathrm{p}=0.6019)$.

Survival. Survival periods after recurrence in the two groups were compared using the Kaplan-Meier method (Fig. 1). The median survival period after recurrence was 11.4 months in the S-1 group and 6.2 months in the GEM/BSC group, with survival in the former being significantly longer than that in the latter ( $\mathrm{p}=0.025)$. The estimated OAS in the $\mathrm{S}-1$ and GEM/BSC groups at 3 years was 24.7 and $7.6 \%$, respectively, again being significantly longer in the former than in the latter $(\mathrm{p}=0.0157)$ (Fig. 2). The median period until progression and the 6-month progression-free survival rate were 5.4 months and $38.5 \%$, respectively (Fig. 3).

Toxicity. The toxicity profiles are shown in Table II. Severe adverse events (grade 3/4) included leukopenia (3.8\%), neutropenia $(7.7 \%)$, anorexia $(3.8 \%)$, and fatigue $(3.8 \%)$. No treatment-related death occurred.

Efficacy of $S-1$ in terms of recurrence pattern. Among the 51 patients studied, 16 suffered relapse with liver or lung metastasis, 10 developed peritoneal dissemination, and 25 had local recurrence. The efficacy of S-1 in terms of the various patterns of recurrence was evaluated (Table III). The median OAS of the patients who developed lung or liver metastasis and peritoneal dissemination was 10.5 and 13.5 months in the S-1 group and 11.6 and 8.7 months in the GEM/BSC group, respectively. A log-rank test using the Kaplan-Meier method revealed significant difference between the two groups. However, the median OAS of the patients who developed local recurrence was 26.9 months in the $\mathrm{S}-1$ group and 17.8 months in the GEM/BSC group $(p=0.0469)$. This result indicates that $\mathrm{S}-1$ was capable of prolonging the OAS in patients who developed local recurrence. 


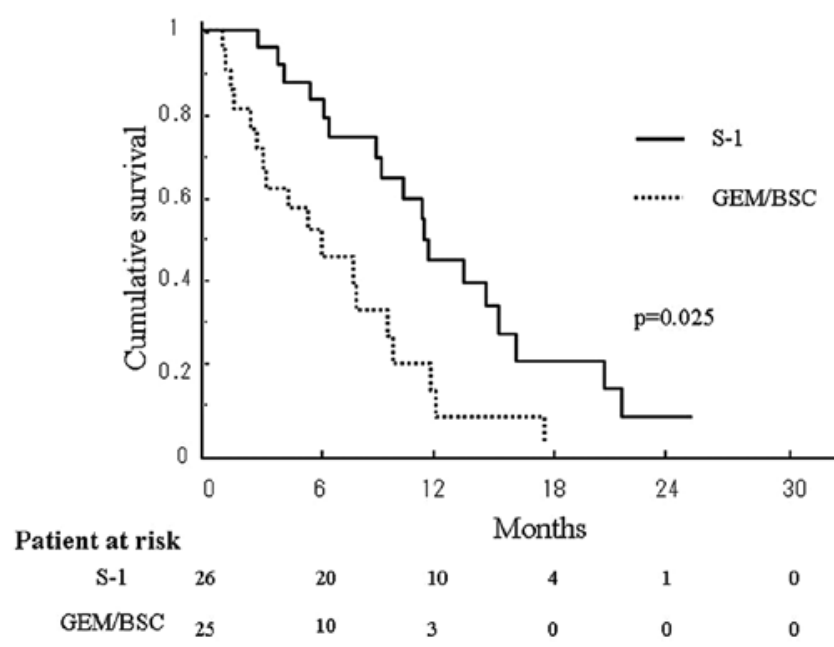

Figure 1. Kaplan-Meier curves for survival periods after recurrence in the S-1 group (solid line) and the GEM/BSC group (dotted line).

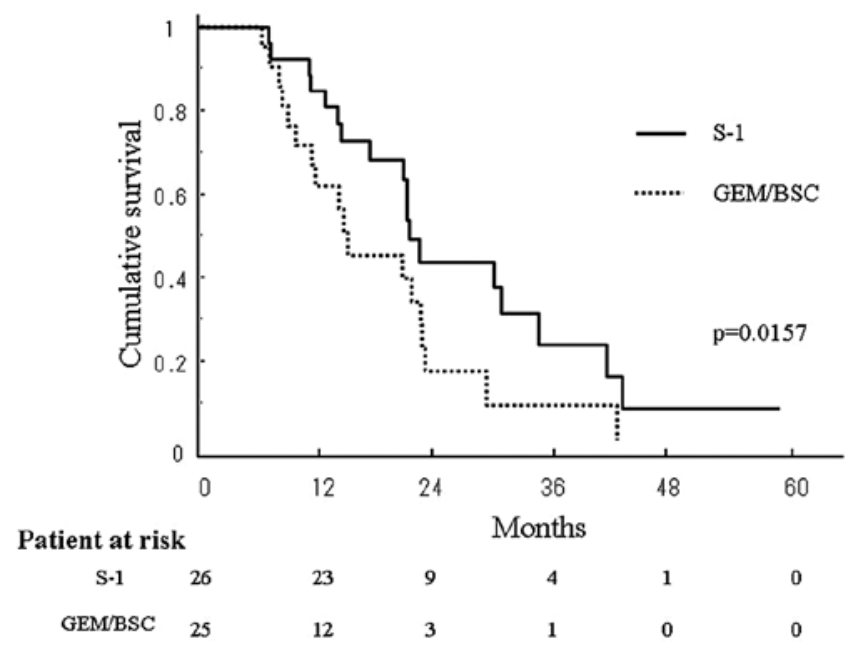

Figure 2. Kaplan-Meier curves for overall survival periods in the S-1 group (solid line) and the GEM/BSC group (dotted line).

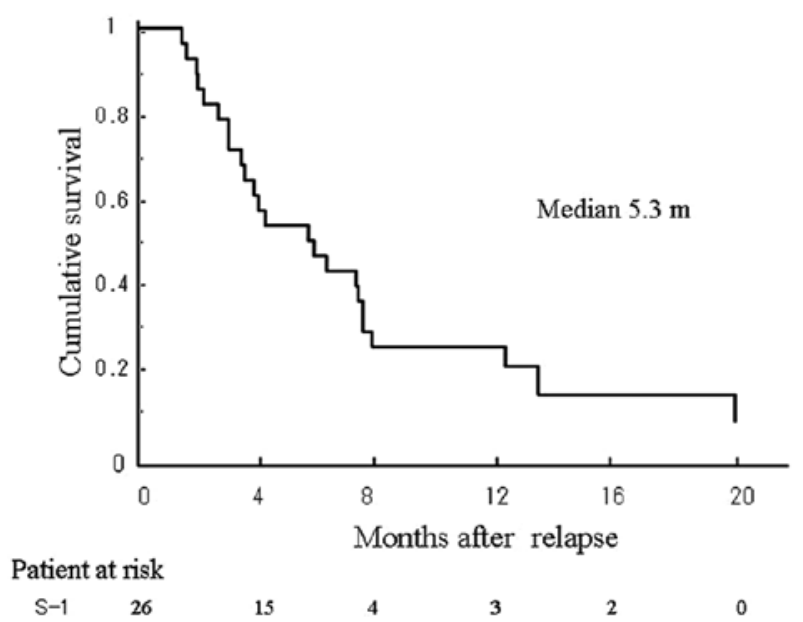

Figure 3. Kaplan-Meier curves for progression-free survival period in the S-1 group. m, months.
Table II. Drug-related adverse effects.

\begin{tabular}{lcc}
\hline & \multicolumn{2}{c}{ S1 group (n=26) } \\
\cline { 2 - 3 } & G1/2(\%) & G3/4 (\%) \\
\hline Hematological toxicity & & \\
Leukopenia & $4(15.4)$ & $1(3.8)$ \\
Neutropenia & $3(11.5)$ & $1(3.8)$ \\
Anemia & $1(0.4)$ & $0(0.0)$ \\
Thrombopenia & $0(0.0)$ & $0(0.0)$ \\
Non-hematological toxicity & & \\
Appetite loss & $2(7.7)$ & $2(7.7)$ \\
Diarrhea & $0(0.0)$ & $0(0.0)$ \\
Nausea & $1(3.8)$ & $0(0.0)$ \\
Vomiting & $3(11.5)$ & $0(0.0)$ \\
Fatigue & $3(11.5)$ & $1(3.8)$ \\
\hline
\end{tabular}

Table III. Efficacy of S-1 in terms of recurrence pattern.

\begin{tabular}{lccc}
\hline & $\begin{array}{c}\text { S-1 } \\
\text { MST } \\
\text { (months) }\end{array}$ & $\begin{array}{c}\text { GEM/BSC } \\
\text { MST } \\
\text { (months) }\end{array}$ & $\begin{array}{c}\text { p-value } \\
\text { (log-rank) }\end{array}$ \\
\hline Liver metastasis & 10.5 & 11.6 & 0.796 \\
Peritoneal dissemination & 13.5 & 8.7 & 0.152 \\
Local recurrence & 26.9 & 17.8 & 0.046 \\
\hline MST, median survival time. & & & \\
\hline
\end{tabular}

\section{Discussion}

In this retrospective study, we investigated the efficacy and feasibility of S-1 as second-line chemotherapy after adjuvant chemotherapy with GEM for patients with pancreatic cancer. Our results show that the administration of S-1 as a secondline chemotherapy was capable of prolonging not only the survival period after relapse (median 11.4 vs. 6.2 months), but also the overall survival period (median 20.9 vs. 13.7 months). Second-line chemotherapy with S-1 combined with adjuvant chemotherapy using GEM may therefore be an efficient and beneficial strategy for pancreatic cancer patients.

Neoptolemos et al previously demonstrated that adjuvant chemotherapy was potentially beneficial for patients with pancreatic cancer, whereas adjuvant chemoradiotherapy had a deleterious effect on survival (6). Tani et al have reported that adjuvant chemotherapy was an independent factor affecting long-term survival in patients with locally advanced pancreatic cancer who had undergone surgery (10). Oettle et al have shown that adjuvant chemotherapy with GEM for pancreatic cancer patients was significantly effective for prolonging disease-free survival (7), and their subsequent study revealed that it was also capable of prolonging OAS (9). In their study, Ueno et al have shown that GEM prolonged diseasefree survival in patients who had undergone macroscopically curative resection of pancreatic cancer (8). Since these reports 
were published, adjuvant chemotherapy with GEM has been the standard treatment in Japan for patients following resection of pancreatic cancer. However, few reports have described the optimal regimens for patients who suffer relapse after adjuvant chemotherapy. In the present study, we retrospectively evaluated the efficacy and safety of S-1, an oral fluoropyrimidine derivative, as second-line chemotherapy for patients suffering disease relapse after adjuvant chemotherapy with GEM.

S-1 is an oral anticancer drug consisting of tegafur, a prodrug of 5-FU, and two biochemical modulators, 5-chloro2,4-dihydroxypyridine and potassium oxonate (11). S-1 has been shown clinically to exert potent antitumor activity against various solid tumors (12-15). Okusaka et al have reported that $\mathrm{S}-1$ is a promising agent for advanced pancreatic cancer, with a response rate of $37.5 \%$ and an MST of 9.2 months (16) In our present study, the MST after recurrence was prolonged for up to 11.4 months by S-1 administration. The median progression-free survival time after administration of S-1 was estimated to be 5.4 months. Results show that second-line chemotherapy with S-1 was capable of maintaining progression-free survival for approximately 6 months, but also extended survival for an additional 6 months. This may have been due to the fact that the toxicity of S-1 was sufficiently mild to allow the introduction of third-line chemotherapy.

In general, S-1 should be administered orally for 28 conse-cutive days, followed by a 14-day rest. However, the incidence of adverse reactions tended to be high (83.2\%), and $20.3 \%$ of all adverse reactions were reported to be of grade 3 or more severe $(12,16)$. Therefore, certain previous reports have proposed that S-1 should be administered for 2 weeks, followed by a 1-week rest, rather than for 4 weeks followed by a 2 -week rest. Tsukuda $e t$ al have reported that, in patients with advanced head and neck cancer, a 2-week administration of S-1 followed by a 1-week rest was safer and more tolerable than 4-week administration followed by a 2-week rest (18). With regard to the administration of S-1 for advanced or recurrent gastric cancer, Kimura et al have reported that the rate of adverse reactions was $77 \%$ in the 2 -week regimen, compared with $93 \%$ for the 4 -week regimen. They also reported that the total 6-month compliance for $\mathrm{S}-1$ was much more favorable for the 2-week regimen than for the 4-week regimen. These authors concluded that the 2-week regimen may mitigate adverse reactions and prolong the medication period (19). In the present study, S-1 was administered orally for 14 consecutive days, followed by a 7-day rest (2-week regimen). Neither hematological nor non-hematological adverse events were frequent. Severe adverse effects (grade 3/4) were almost not evident, and the medication time was therefore prolonged. This may have contributed to prolonging not only progression-free but also overall survival.

S-1 administration was not capable of prolonging the OAS of patients who had suffered relapse in the form of either peritoneal dissemination or liver or lung metastasis, and was effective only for local recurrence. S-1 administration allowed patients who had suffered local recurrence to survive longer than those who continued with GEM, or received best supportive care. In a phase II study report, Okusaka et al stated that S-1 administration was effective against metastatic pancreatic cancer. In their study, although $90 \%$ of patients had liver metastasis, a relatively long MST (9.3 months) was observed (16). In the present study, as only a small number of patients developed relapse in the form of liver metastasis, the effectiveness of S-1 may not have reached a significant level.

In conclusion, following not only major surgical treatment, but also cancer relapse, patients experience a relatively severe condition. S-1, an oral anticancer drug, is capable of maintaining a reasonable quality of life under such conditions (20). Since this study revealed a promising anticancer effect of S-1 and a significantly long survival time, S-1 is a potentially beneficial drug for second-line chemotherapy following adjuvant chemotherapy with GEM in patients with pancreatic cancer.

\section{References}

1. Jemal A, Siegel R, Ward E, Hao Y, Xu J, Murray T and Thun MJ: Cancer statistics. CA Cancer J Clin 58: 71-96, 2008.

2. Bradley EL: Long-term survival after pancreatoduodenectomy for ductal adenocarcinoma: the emperor has no clothes? Pancreas 37: 349-351, 2008.

3. Yeo CJ, Cameron JL, Lillemoe KD, et al: Pancreaticoduodenectomy for cancer of the head of pancreas: 201 patients. Ann Surg 221: 721-733, 1995.

4. Neoptolemos JP, Dunn AA, Stocken DD, et al: Adjuvant chemoradiotherapy and chemotherapy in resectable pancreatic cancer: a randomized controlled trial. Lancet 358: 1576-1585, 2001.

5. Ueno H, Kosuge T, Matsuyama Y, Yamamoto J, Nakao A, Egawa S, Doi R, Monden M, Hotori T, Tanaka M, Shimada M and Kanemitsu K: A randomised phase III trial comparing gemcitabine with surgery-only in patients with resected pancreatic cancer. Japanese Study Group of Adjuvant Therapy for pancreatic cancer. Br J Cancer 101: 908-915, 2009.

6. Neoptolemos P, Stocken DD, Friess H, et al: A randomized trial of chemoradiation therapy and chemotherapy after resection of pancreatic cancer. N Engl J Med 350: 1200-1210, 2004.

7. Oettle H, Post S, Neuhaus P, et al: Adjuvant chemotherapy with gemcitabine vs observation in patients undergoing curativeintent resection of pancreatic cancer: a randomized controlled trial. JAMA 297: 267-277, 2007.

8. Ueno H, Okusaka T, Ikeda M, Takezako Y and Morizane C: Phase II study of S-1 in patients with advanced biliary tract cancer. Br J Cancer 91: 1769-1774, 2004.

9. Neuhaus P, Riess H, Post S, et al: CONKO-001: Final results of the randomized, prospective, multicenter phase III trial of adjuvant chemotherapy with gemcitabine versus observation in patients with resected pancreatic cancer (PC). J Clin Oncol 26: 214, 2008 (suppl; abstr LBA4504).

10. Tani M, Kawai M, Terasawa H, Ina S, et al: Prognostic factor for long-term survival in patients with locally invasive pancreatic cancer. J Hepatobiliary Pancreat Surg 14: 545-550, 2007.

11. Shirasaka T, Shimamoto Y, Ohshimo H, et al: Development of a novel form of an oral 5-fluorouracil derivative (S-1) directed to the potentiation of the tumor selective cytotoxity of 5-fluorouracil by two biochemical modulators. Anticancer Drugs 7: 548-557, 1996.

12. Sakata Y, Ohtsu A, Horikkoshi N, Sugimachi K, Mitachi Y and Taguchi T: Late phase II study of novel oral fluoropyrimidine anticancer drug S-1 (1M tegafur- $0.4 \mathrm{M}$ gimestat-1M otastat potassium) in advanced gastric cancer patients. Eur J Cancer 34: 1715-1720, 1998.

13. Saeki T, Takashima S, Sano M, et al: A phase II study of S-1 Cooperative Study breast cancer - a Japanese trial by the S-1 Cooperative Study Group, Breast Cancer Working Group. Breast Cancer 11: 194-202, 2004.

14. Fukushima M, Satake H, Uchida J, et al: Preclinical antitumor efficacy of S-1: A new oral formulation of 5-fluorouracil on human tumor xenografts. Int J Oncol 13: 693-698, 1998.

15. Ueno H, Okusaka T, Ikeda M, Takezako Y and Morizane C: An early phase II study of S-1 in patients with metastatic pancreatic cancer. Oncology 68: 171-178, 2005. 
16. Okusaka T, Funakoshi A, Furuse J, Boku N, Yamao K, Ohkawa S and Saito H: A late phase II study of S-1 for metastatic pancreatic cancer. Cancer Chemother Pharmacol 61: 615-621, 2008.

17. Sugimachi K, Maehara Y, Horikoshi N, et al: An early phase II study of oral S-1, a newly developed 5-fluorouracil derivatives for advanced and recurrent gastrointestinal cancers. Oncology 57: 202-210, 1999

18. Tsukuda M, Kida A, Kono N, Yoshihara T, Hasegawa Y, Sugita M and Chemotherapy Study Group of Head and Neck Cancer: Randomized scheduling feasibility study of S-1 for adjuvant chemotherapy in advanced head and neck cancer. Br J Cancer 93: 884-889, 2005.
19. Kimura Y, Kikkawa N, Iijima S, et al: A new regimen for S-1 therapy aiming at adverse reaction mitigation and prolonged medication by introducing a 1-week drug-free interval after each 2-week dosing session: efficacy and feasibility in clinical practice. Gastric Cancer 6: 34-39, 2003.

20. O'Neill VJ and Twelves CJ: Oral cancer treatment: developments in chemotherapy and beyond. Br J Cancer 87: 933-937, 2002. 